\title{
XXXI. Krystallographische Untersuchung einiger Derivate der Terephtalsäure.
}

\author{
Von \\ Wilh. Muthmenn in Worcester, Mass.
}

(Mit 22 Holzschnitten.)

In seinen Arbeiten uber die Constitution des Benzols*) hat A. v. B a e yer eine grosse Anzahl von Derivaten der Terephtalsäure beschrieben, welche theils durch directe Reduction der Säure mit Natriumamalgam, theils durch Addition von Brom und Bromwasserstoff, sowie durch Substitution von Brom aus diesen Reductionsproducten erhalten worden waren. Herr Prof. v. Baeyer hatte die Gute, mir diejenigen der von ihm erhaltenen Producte, welche eine Messung ermöglichten, zur krystallographischen Bestimmung zu thbergeben, welche nicht nur fur den Chemiker von Interesse sein durfte - indem sie ein bequemes Mittel zur Identificirung und Wiedererkennung der beschriebenen Körper liefert - , sondern auch für den Krystallographen von Werth ist, da hier eine schöne zusammenhängende Reihe chemisch verwandter Substanzen vorliegt. In der That haben sich auch mehrere morphotropische Beziehungen und Einblicke in den Zusammenhang zwischen Krystallform und chemischer Constitution ergeben; ich bin daher meinem verehrten Lehrer zu grossem Danke verplichtet für die liebenswürdige Bereitwilligkeit, mit der er die Arbeit durch Ueberlassung des theilweise sehr schwierig zu beschaffenden Materials förderte, und war es mir eine angenehme Pllicht, ihm in einzelnen Fällen durch Identificirung seine Untersuchung erleichtern zu können.

Die Messungen wurden im Laboratorium der mineralogischen Staatssammlung zu Munchen ausgefuhrt.

*) Liebig's Ann. d. Chem. 1888, 245, 103 und ebenda 1889, 251, 257. 


\section{Terephtalsäuredimethylester.}

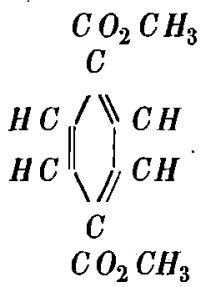

Schmelzpunkt 1400. Zuerst dargestellt von de 1 a $R$ ue und $M$ üller.

Lieb. Ann. d. Chem. 121, 89. Siehe auch A, v. B a eyer, ebenda 1888, 245, 140.

Krystallsystem: Rhombisch.

$a: b: c=0,84281: 1: 3,0826$.

Fig. 1.

Beobachtete Formen: $c=\{001\} 0 P, b=$ $\{010\} \infty \breve{P}_{\infty}, \quad 0=\{111\} P$. Dunne Tafeln nach der Basis, meistens in der Richtung der $a$-Axe bedeutend verlängert (Fig. 1).

$$
\begin{aligned}
& \text { Gemessen: Berechnet } \\
& o: o=(111):(1 T 1)=78^{\circ} 13^{\prime} \\
& o: 0=(111):(\text { TT1 })=15623 \\
& 0: 0=(1 / 1):(\bar{T} 11)=9650
\end{aligned}
$$

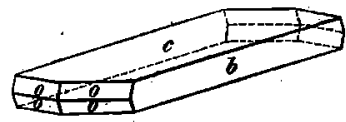

Farblos, durchsichtig. Die optische Axenebene ist dem Brachypinakoid parallel. Durch (001) siebt man keine Axen austreten.

\section{2. $\Delta^{1,4}$-Dihydroterephtalsäuredimethylester.}

$$
\begin{aligned}
& \mathrm{CO}_{2} \mathrm{CH}_{3}
\end{aligned}
$$

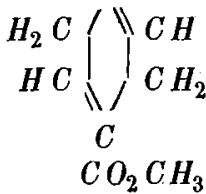

Schmelzpunkt 1300. Krystalle aus Essigäther.

Fig. 2.

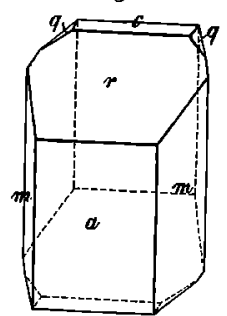

Fig. 3.

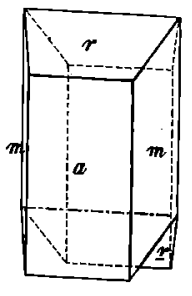

$\{012\} \frac{1}{2} R \infty, c=\{001\} 0 P$ (Fig. 2). $\quad\{012\}$ wurde nur einmal beobachtet, weshalb als durchaus zuverlässig nur die beiden ersten in untenstehender Tabelle angegebenen Winkel zu betrachten sind. Die Basis tritt nur selten auf und 
waren die Krystalle, an denen diese Form beobachlel wurde, schlecht ausgebildet und gaben keine hinreichend guten Bilder am Goniometer; wabrscheinlich enthielten sie noch Terephtalsäureester als Verunreinigung. Häufig Zwillinge nach (100) (s. Fig. 3 auf S. 461).

$$
\begin{array}{llc}
\text { Gemessen : } & \text { Berechnet: } \\
a: m=(100):(110)={ }^{*} 69031^{\prime} & - \\
a: r=(100):(101)={ }^{*} 49 & 17 & - \\
r: q=(101):(012)={ }^{*} 43 & 21 & - \\
m: r=(110):(101)= & 7648 & 76048^{\prime} \\
m: q=(110):(012)=5059 & 5331 \\
m: q=(110):(012)=6112 & 616 \\
a: q=(100):(012)=7748 & 7725
\end{array}
$$

Farblos, durchsichlig. Durch (100) siebt man im Polarisationsinstrumente eine Axe austreten, die optische Axenebene ist der Symmetrieebene, parallel. Doppelbrechung ziemlich schwach. Die Zwillinge sind meist nur optisch als solche zu erkennen und gleichen, da sie fast immer an einem Ende aufgewachsen sind, in ibrer geometrischen Ausbildung genau rhombischen Krystallen.

\section{3. $\Delta^{1,3}$.Dihydroterephtalsäuredimethylester.}

$$
\begin{gathered}
\mathrm{CO}_{2} \mathrm{CH}_{3} \\
\mathrm{H}_{2} \mathrm{C} / \prod_{\mathrm{H}_{2} \mathrm{CH}} \bigvee_{\mathrm{CH}}^{\mathrm{CH}} \\
\mathrm{C} \mathrm{O}_{2} \mathrm{CH}_{3}
\end{gathered}
$$

Schmelzpunkt 850. Krystalle aus Ligroin.

Krystallsystem: Monos y metrisch.

$$
a: b: c=2,2408: 1: 3,5907 ; \quad \beta=87013^{\prime} .
$$

Fig. 4.

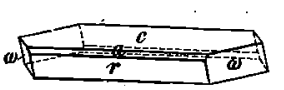

Beobachtete Formen : $a=\{100\} \infty \mathbb{2} \infty, c=$ $\{001\} 0 P, r=\{T 01\}+P \infty, \omega=\{T 11\}+P$. Tafeln nach der Basis; $\{100\}$ tritt meist nur ganz schmal auf (Fig. 4).

$$
\begin{aligned}
& \text { Gemessen: Berechnet: } \\
& a: r=(T 00):(T 01)={ }^{*} 32044^{\prime} \\
& a: c=(100):(001) \doteq{ }^{*} 8713 \\
& r: \omega=(T 01):(T 11)={ }^{*} 6245 \\
& a: \omega=(\bar{T} 00):(\bar{T} 11)=6729 \\
& 670 \mathrm{z \prime} \\
& c: \omega=(001):(T 11)=76.51 \\
& 76 \quad 47
\end{aligned}
$$


Die Krystalle werden beim Liegen an der Luft schon nach kurzer Zeit tribe und undurchsichtig.

Farblos; durch die Basis tritt fast genau senkrecht eine Bisectrix und zwar die stumpfe aus, da selbst im Sch n e i der'schen Axenwinkelapparate keine Axen gesehen wurden. Optische Axenebene ist die Symmetrieebene.

Doppelbrechung sehr stark.

Die drei oben beschriebenen Körper vermögen zu Mischkrystallen zusammenzutreten, obwohl sie in Bezug auf die geometrischen Formen ihrer Krystalle keineswegs als isomorph bezeichnet werden können. Zwar zeigt sich in einigen Winkeln eine unverkennbare Aehnlichkeit; namentlich gilt dies für die Neigung der pyramidalen Formen gegen die vorherrschende Fläche der Orthozone resp. gegen die Basis, wie aus folgender Zusammenstellung hervorgeht:

$$
\begin{aligned}
& \text { Terephtalsäureester } \quad(001):(111)=780111^{\prime} \\
& \Delta^{1,4} \text {-Dihydroester } \quad\left\{\begin{array}{l}
(101):(110)=7648 \\
(100):(012)=77 \quad 48
\end{array}\right. \\
& \Delta^{1,3} \text {-Dihydroester } \quad(001):(\overline{1} 11)=76 \quad 47
\end{aligned}
$$

Die beiden Dihydroester haben auch einen Winkel in der Orthozone nabe ubereinstimmend:

$$
\begin{array}{ll}
\Delta^{1,4} \text {-Dihydroester } & (001):(\bar{T} 01)=32017^{\prime} \\
\Delta^{1,3} \text {-Dihydroester } & (\bar{T} 00):(\bar{T} 01)=3244
\end{array}
$$

doch muss dazu bemerkt werden, dass bei dem $A^{1,4}$-Ester die Basis und das hintere Hemidoma niemals an Krystallen der reinen Substanz beobachtet wurden. Endlich ist noch in optischer Beziehung eine Analogie zu verzeichnen, durch die Basis des monosymmetrisch krystallisirenden $\Delta^{1,3}-\mathrm{Di}$ hydroesters tritt fast genau senkrecht die stumpfe Bisectrix aus, bei dem rhombischen Terephtalsăureesster ist die zweite Mittellinie senkrecht zu dem vorherrschenden (001) und bei dem $\Delta^{1,4}$-Dihydroestep liegt eine Bisectrix ebenfalls fast vertical zu einer Querfläche, nämlich zum vorderen Hemidoma.

Trotz dieser morphotropischen Beziehungen kann, wie gesagt, von Isomorphie keine Rede sein, und es ist daher das Zusammenkrystallisiren der drei Körper eine sehr auffallende Thatsache, die an die analogen Erscheinungen beim Succinylobernsteinsäureester und $p$-Dioxyterephtalsäureester, sowie beim Diinid des Succinylobernsteinsäureesters und dem Diamidoterephtalsăureester erinnert. Ich habe es aus diesem Grunde schon früher als wahrscheinlich hingestellt*), dass der Succinylobernsteinsäure-

*) Diese Zeilscbr. 1888, 15, 77. 
ester aufzufassen sei als $p$-Dioxydihydroterephtalsäureester, eine Schlussfolgerung, die durch die neueren chemischen Forschungen über diesen Körper mehr und mehr bestätigt wird.

Dass der Terephtalsäureester und der $\not^{1,4}$-Dihydroester zusammenkrystallisiren; beobachtete v. B a y er, welcher diese beiden Substanzen durch Umkrystallisiren nicht zu trennen vermochte. Die von mir untersuchten Krystalle bestanden zum grössten Theile aus Dihydroester und enthielten nicht mehr als höchslens $5 \%$ Terephtalsäureester. Sie zeigten den Habitus und die Formen der ersteren Substanz, doch traten ausser den an den reinen Krystallen derselben beobachteten Flächen noch (001) und (T01) auf. Sie waren in ihrer Ausbildung gestört und gaben bei der Messung sehr schwankende Werthe; es würde an denselben gefunden:

\begin{tabular}{|c|c|c|}
\hline$(100):(001)=$ & $\begin{array}{l}\text { Miscbkrystalle: } \\
\text { ca. } 71 \frac{1}{2}{ }^{0}-73 \frac{1}{2}^{0}\end{array}$ & $\begin{array}{c}\text { Reiner } 12,5 \text {-Dihydroester : } \\
\qquad 4^{0} 14^{\prime}\end{array}$ \\
\hline$(001):(T 01)=$ & $30^{0} 28^{\prime}$ & 3244 \\
\hline$(100):(101)=$ & $47^{0} 38^{\prime}-49^{0} 46^{\prime}$ & $49 \quad 17$ \\
\hline$(100):(110)=$ & $\begin{array}{llll}68 & 21 & -69 & 39\end{array}$ & 6931 \\
\hline
\end{tabular}

Die Mischung wurde sowohl aus Holzgeist, als auch aus Schwèfelkohlenstoff umkrystallisirt, doch resultirten wieder Mischkrystalle mit den oben beschriebenen Unregelmässigkeiten in der Ausbildung, die immer elwas trube erschienen. Dagegen gaben die mir von Herrn Prof. v. B a e yer zur Verfiugung gestellten Proben von reinem $\mathbf{4}^{1,4}$-Dihydroester aus den genannten Lösungsmitteln sowohl als auch aus Essigäther prächtig ausgebildete, schön durchsichtige Krystalle, die auf dem Goniometer gute Reflexe und bei der Messung bis auf wenige Minuten übereinstimmende Winkelwerthe zeigten.

Man sieht aus diesem Beispiele, dass die Krystallographie dem Chemiker nicht nur durch Identificirung nützlich sein kann, sondern demselben manchmal auch gute Dienste zu leisten vermag, wenn es sich darum handelt zu entșcheiden, ob eine einheitliche Substanz wirklich ein chemisches Individuum repräsentirt oder eine Mischung zweier nahe verwandter Körper ist. Ich habe auf diesen Punkt schon früher aufmerksam gemacht; auch die Mischkrystalle von Schwefel und Selen zeigen solche, "geometrische Anomalien «, bestehend in einer durch Nichtparallelität der zueinander gehörenden Flächen hervorgerufenen Schwankung in den Winkelwerthen; bei Mischkrystallen von Diamidoterephtalsäureester und dem Diimid des Succinylobernsteinsäureesters nach der Form des ersteren Körpers beobachtete ich Abweichungen bis zu $8^{\circ}$ an Winkeln, welche nach den Symmetrieverhältnissen der Componenten hätten gleich sein müssen, und im Verlaufe dièser Mittheilung werde ich noch mehrfach Gelegenheit haben, ähnliche Erscheinungen zu erwähnen. Es scheint mir aus alledem 
hervorvorzugehen, dass die Unregelmässigkeiten in der geometrischen Ausbildung der Krystalle mehr Aufmerksamkeit verdienen, als man denselben bisher zugewendet hat.

Der $\Delta^{1,4}$ - und der $\Delta^{1,3}$-Dihydroäther vermögen in allen Verhältnissen zusammenzukrystallisiren; ich schliesse dies aus den Schmelzpunkten der von mir aus gemischten Lösungen erhaltenen Producte, die von $82^{\circ}-125^{0}$ variirten, wäbrend der reine $A^{1,3}$-Ester bei $85^{0}$, der reine $\Delta^{1,4}$-Ester bei $130^{\circ}$ schmilzt. Einen weiteren Beweis dafür, dass ich Mischkrystalle unter den Händen hatle, lieferte mir das Verhalten der Substanzen bei der Sublimation; wurde diese sorgfältig zwischen zwei Uhrgläsern ausgefuhrt, so sublimirte zunächst $\Delta^{1,3}$-Ester, während der schwerer flüchtige $\Delta^{1,4}$-Ester zurückblieb.

Ich habe Mischkrystalle sowohl nach der Form des $\mathbf{1}^{1,4}$-Esters als auch nach der des $\Delta^{1,3}$-Esters beobachtet. Die letztere Form zeigten die Producte, wenn die Substanz auf dem Wasserbade schmolz, während die Krystalle nach der ersteren Form einen Schmelzpunkt uber $100^{\circ}$ hatten. In einer viel $\Delta^{1,3}$-Ester enthaltenden Krystallisation aus Essigäther wurde an drei Krystallen der Schmelzpunkt bestimmt; ich fand 820, $83^{\circ}$ und 870. Die Messung dieser Krystalle gab folgende Werthe:

$\begin{array}{lc}\text { Mischkrystalle: } & \text { Reiner } \triangle 1,3 \text {-Aether : } \\ (T 00):(T 01)=\quad 31^{\prime} 10^{\prime} & 32^{0} 44^{\prime} \\ (100):(001)=86^{0} 33^{\prime}-88^{\circ} 5^{\prime} & 8713 \\ (T 01):(T 11)=6236-6242 . & 6245 \\ (001):(T 11)=7558-7747 & 7647\end{array}$

Das Mittel dieser Zahlen stimmt also ziemlich gut mit den an den Krystallen des reinen $\Delta^{1,3}$-Esters gefundenen Werthen; doch zeigten die Misçhkrystalle ebenfalls wieder die oben erwähnten, geometrischen Anomalien. So wurde an einem Exemplare, dessen Flächen gute und einheitliche Reflexe gaben, und das schön durchsichtig war, der WinkeI $(001):(111)=76^{0} 1^{\prime}$ und (001):(TT1) $=77025$ ' bestimmt; auch die optischen Eigenschaften liessen erkennen, dass keine einheitliche Substanz vorlag, denn das Lemniscatensystem war gestört und die stumpfe Bisectrix war nicht wie bei den reinen Krystallen des $\Delta^{1,3}$-Esters auf $(001)$ senkrecht, sondern bildete einen kleinen Winkel, etwa $5^{0}$ mit der Normalen zu dieser Fläche. Was den Habitus anbetrifft, so waren die Krystalle ganz so wie die der reinen Substanz ausgebildet.

Anders ist es in dieser Beziehung mit den Mischkrystallen zweiter Art nach der Form des $\mathbf{A}^{1,4}$-Esters. Während die reinen Krystalle hier immer tafelförmig nach dem Orthopinakoid ausgebildet und nach der Verticalaxe verlängert sind, herrschte an den Mischkrystallen das Hemidoma $\{101\}$ vor, 
und waren dieselben nach der Symmetrieaxe verlängert. Sie zeigten nur die Formen $\{101\},\{100\}$ und $\{001\}$; die Winkelmessungen ergaben:

$\begin{array}{ccc}\text { Mischkrystalle: } & \text { Reine Substanz: } \\ (100):(110)=67036^{\prime}-69^{\circ} 41^{\prime} & 69^{\circ} 31^{\prime} \\ (100):(101)=4914-5017 & 4917 \\ (110):(101)=7622-7728 & 7648\end{array}$

Die optischen Eigenschaften zeigten sich vielfach gestört; nicht nur dass die Bisectrix zur Normalen auf (101) manchmal geneigt erschien, die Axenebene war auch gegen die Symmetrieebene oft gedreht. Da zuweilen ein Prismenflächenpaar ganz fehlte, so präsentirten sich manche der Krystalle als asymmetrische, von drei Flächenpaaren begrenzte Tafeln.

Der Schmelzpunkt dieser Mischungen war sehr unscharf. Die erste Probe begann bei $105^{0}$ und war bei $114^{0}$ ganz geschmolzen; eine zweite zeigte $10^{0}-111^{0}$; von einer dritten endlich verflussigte sich ein Theil schon bei $82^{0}$; in der geschmolzenen Masse blieben jedoch feste Partikeln, die bei $105^{\circ}$ anfingen zu schmelzen und bei $110^{\circ}$ verschwunden waren.

Aus den oben mitgetheilten Thatsachen geht hervor, dass die beiden beschriebenen Ester sich bei der Bildung von Mischkrystallen gerade so verhalten, wie es bei einer Anzahl von anderen chemisch verwandten Substanzen beobachtet worden ist; die Mischungen zeigen, abgesehen von geometrischen Anomalien, die Form, in welcher der in ihnen uberwiegende Gemengtheil fur sich krystallisirt. Dabei liegt kein Grund vor, eine Isodimorphie anzunehmen; im Gegentheil, dies ist mir im vorliegenden Falle sogar unwahrscheinlich. Ich erkläre mir die in Rede stehenden Thatsachen folgendermassen :

In einem Raumgitter wirken die einzelnen Molekule nicht nur auf die ihnen direct benachbarten, sondern die Anziehung erstreckt sich auch auf entfernter liegende Punkte. Denken wir uns nun in einem solchen Gefuge einen Krystallbaustein durch einen ähnlichen ersetzt, der in etwas anderer Weise und in anderen Richtungen wirkt, so wird derselbe zwar eine Störung hervorrufen, allein im Grosșen und Ganzen wird die Structur nicht verändert werden. Von solchen fremdartigen Molekeln wird der Krystall in mehr oder weniger regelmässig sich wiederholenden Abständen mehrere in sich aufnehmen können, ohne dass die Form und die Dimensionen der Elementarparallelepipeda, resp. die durch diese beiden Factoren bedingte aussere Begrenzung des Krystalls, seine geometrische Form, wes entlich beeinflusst werden; die fremden Molekule werden gewissermassen gezwungen, sich den ubrigen zu accommodiren. Im Allgemeinen giebt es natürlich eine Grenze im Verhältnisse der beiden Subștanzen, die nicht uberschritten werden kann und die von der grösseren oder geringeren Aehnitlichke der beiden Kérper abhăngt; auch wird die Form des Mischkrystalles 
nicht mehr genau der der Krystalle des vorherrschenden Gemengtheiles entsprechen, sondern gestört sein. Wichtig ist, dass diese Abweichungen von der Idealform meist keine Regelmässigkeiten zeigen; sie documentiren sich auf verschiedene Weise. Hatten sich die Krystalle schnell abgeschieden, so sind die Flächen gebrochen und geben mehrfache Bilder am Goniometer; erfolgte die Ausbildung langsam und stetig, so zeigen die Winkel unregelmässige Abweichungen; sie sind bald grösser bald kleiner als an den reinen Krystallen. Charakteristisch ist auch die Nichtparallelität der zueinander gehörigen Flächen, die oft einen Winkel bis zu $5^{0}$ mit einander bilden.

\section{4. $A^{2,5}$.Dihydroterephtalsäure.}

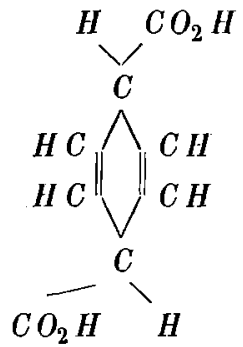

Krystalle aus Essigäther.

Krystallsystem : Mo nos y mmetris ch.

$$
a: b: c=0,98203: 1: 1,0188 ; \beta=76^{0} 2^{\prime} \text {. }
$$

Fig. 5.

Beobachtete Formen: $m=\{110\} \infty P, c=\{001\} 0 P, r=$ $\{\overline{1} 01\}+\mathbb{P} \infty$. Nach der $c$-Axe gestreckt (Fig. 5). Die Flächen der kleinen Kryställchen waren in ihrer Ausbildung vielfach gestört und gestreift und gaben nur schlechte Bilder am Goniometer.

$$
\begin{array}{lccccc} 
& \text { Zahl der } & \text { Messungen: } & \text { Grenzwerthe : } & \text { Mittel : } & \text { Berechnet: } \\
m: m=(110):(1 T 0) & 20 & 85^{0} 17^{\prime}-88^{0} 47^{\prime} & * 87^{\prime} 14^{\prime} & - \\
m: c=(110):(001) & 10 & 7840-8030 & * 7956 & - \\
c: r=(001):(T 01) & 5 & 5255-5410 & * 5320 & - \\
m: r=(T 10):(T 01) & 6 & 59.31-6213 & 61 & 0 & 62040^{\prime}
\end{array}
$$

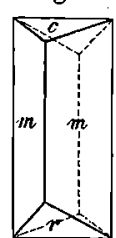

Farblos, durchsichtig. Die Auslöschungsrichlung auf (110) bildet einen Winkel von etwa $18^{\circ}$ mit den Prismenkanten. Zur näheren optischen Untersuchung eigneten sich die Krystalle nicht. 


\section{5. $A^{1}$-Tetrahydroterephtalsäurediphenylester.}

$$
\begin{aligned}
& \mathrm{CO}_{2} \mathrm{C}_{6} \mathrm{H}_{5} \\
& \text { C }
\end{aligned}
$$

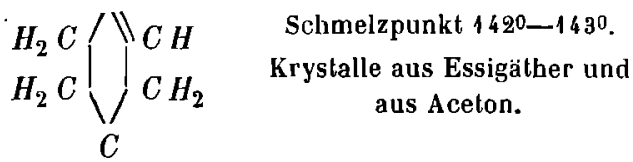

$$
\begin{aligned}
& \mathrm{HCO}_{2} \mathrm{C}_{6} \mathrm{H}_{5}
\end{aligned}
$$

Krystallsystem : Monos y m met ris ch.

$$
a: b: c=2,8244: 1: 2,4702 ; \beta=82^{0} 33^{\prime} \text {. }
$$

Beobacbtete Formen: $a=\{100\} \infty \mathbb{P} \infty, c=\{001\} 0 P, m=\{110\} \infty P$, $r=\{101\}+ \pm \infty, n=\{210\} \infty \pm 2$.

Fig. 6.

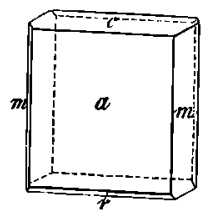

Fig. 7.

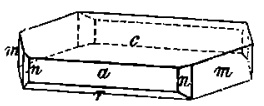

Zwei sehr verschiedene Arten der Ausbildung; Tafeln nach dem Orthopinakoid (Fig. 6) und Tafeln nach der Basis (Fig. 7). $\{210\}$ und $\{101\}$ treten selten und nur schmal auf. $\{001\}$ ist selten gut ausgebildet; bei .den Krystallen vom ersten Typus, welche bessere Reflexe gaben, ist diese Fläche meist einseitig gekrtimmt, was auf eine Hemimorphie schliessen lässt.

Gemessen : Berechnet:

$$
\begin{aligned}
& a: c=(100):(001)={ }^{*} 82033^{\prime} \\
& a: m=(100):(110)={ }^{*} 19021 \\
& a: r=(\bar{T} 00):(T 01)={ }^{*} 33 \\
& m: c=(110):(001)=8734 \quad 87030^{\prime} \\
& a: n=(100):(210)=5430 \quad 5428 \\
& c: n=(100):(210)=8558 \quad 8541
\end{aligned}
$$

Farblos, durchsichtig. Die optische Axenebene ist der Symmetrieebene parallel, durch (001) tritt eine optische Axe unter einem Winkel von etwa $35^{0}$ aus. Doppelbrechung stark.

\section{Amid der $\Delta^{2}$-Tetrahydroterephtalsäure.}

$$
\text { Krystalle aus Wasser. }
$$

Krystallsystem: T etrag o nal.

$$
a: c=1: 2,1505 \text {. }
$$


Beobachlete Formen: $a=\{100\} \infty P \infty, o=\{111\} P$. Sehr dunne Nädelchen nach $a$ (Fig. 8); die Messung der Endflächen war wegen der Kleinheit der Krystalle nur schwer durchfuhrbar.

$o: o=(111):(\bar{T} 11)=84^{\theta} 24^{\prime}$ (Mittel aus 15 Messungen, die zwischen $83^{\circ} 40^{\prime}$ und $86^{\circ} 0^{\prime}$ schwanken).

Farblos, durchsichtig. Doppelbrechung mittel; sehr vollkommen spaltbar nach der Basis; durch solche Spaltblättchen sieht man manchmal das einaxige Kreuz, meist jedoch in Folge optischer Anomalien eine zweiaxige Interferenzfigur mit kleinem Axenwinkel.

Von dem Amid der $\Delta^{1}$-Tetrahydroterephtalsäure erhielt ich von Herrn Prof. v. B a yer gleichfalls eine Probe zur Untersuchung; indessen waren die Krystalle zu klein, als dass eine Messung möglich gewesen wäre. Durch mikroskopische Beobachtungen konnte constatirt werden, dass die Substanz rhombisch, und zwar sphenoidisch-hemiëdrisch krystallisirt. Die Krystalle zeigten eine Combination des Prismas, mit einem Sphenoëder; durch die Prismenflächen tritt fast senkrecht eine optische Axe aus, so dass man in Folge conischer Refraction auf denselben keine Auslöschung bemerkt. Diese Eigenschaft bildet ein gutes Mittel, um die beiden Tetrahydroterephtalsäuren von einander zu unterscheiden, denn das tetragonal krystallisirende Amid der $\Delta^{2}$-Säure zeigt selbstverständlich gerade Auslöschung auf den Prismenflächen.

Oben habe ich erwähnt, dass eine einseitige Rundung auf den Basisflächen des Phenylesters der $\boldsymbol{A}^{1}$-Tetrahydroterephtalsäure auf eine Hemimorphie schliessen lässt; das Amid derselben Säure ist, wie wir sahen, rhombisch-sphenoidjsch-hemiëdrisch. Diese Thatsache ist für die Theorie insofern von grösster Wichtigkeit, als von einer ganzen Anzahl der von v. Baeyer entdeckten bydrirten Terephtalsäuren zwei enantiomorphe Formen möglich sind. Das einfachste Beispiel ist die $\Delta^{1}$-Tetrabydrosäure; denkt man sich den Benzolring in einer wagerechten Ebene und das Carboxyl an dem reducirten Kohlenstoffatome an der oberen Seite des Ringes, so lehrt eine einfache Ueberlegung, dass es einen Unterschied macht, ob die doppelte Bindung sich an der rechten oder linken Seite des nicht reducirten carboxylirten Kohlenstoffatomes befindet. Chemisch wird sich dieser Unterschied nicht nachweisen lassen, wohl aber muss er sich an gewissen physikalischen Eigenschaften der Krystalle zeigen. Bis jetzt habe ich nur die Thatsache constatiren können, dass die beiden untersuchten Derivate der $\boldsymbol{\Delta}^{1}$-Tetrahydroterepthalsäure mit der Theorie übereinstimmen und liefert dies Resultat ein wichtiges Argument für die von v. Baeyer aufgestellte Theorie uber die räumliche Lagerung der Atome im Molekul der 
reducirten Benzolderivate. Ich gedenke die Sache auch in Bezug auf das optische Verhalten der Lösungen weiter zu verfolgen.

\section{Hexahydroterephtalsäure.}

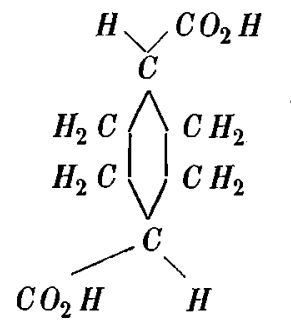

Krystalle aus Aceton.

Krystallsystem: Monos y m me tri s ch.

$$
a: b: c=1,1888: 4: 0,6963 ; \quad \beta=72042 \text {. }
$$

Beobachlete Formen: $a=\{100\} \infty P \infty, b=\{010\} \infty R \infty, c=\{001\} 0 P$, $m=\{110\} \infty P, \omega=\{T 11\}+P$.

Fig. 9.

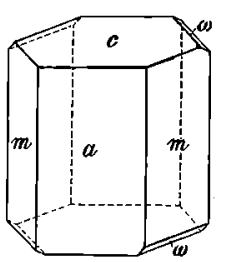

Dicke Tafeln nach dem Orthopinakoid (Fig. 9), doch ist gerade diese Fläche immer sehr stark gekrümmt, so dass eine Messung derselben gänzlich unmöglich war. Die Prismenflächen waren an sämmtlichen Krystallen in der Mitte quer gebrochen und hestanden eigentlich aus zwei Flächen, die einen Winkel von $0^{\circ} 50^{\prime}-1010^{\prime}$ mit einander bildeten. Da dieser Winkel uberall ein ausspringender ist, so liegt eine Zwillingsbildung nicht vor, was uberdies auch die optischen Eigenschaften der Substanz beweisen.

$$
\begin{array}{lc}
\text { Gemessen: } & \text { Berechnet: } \\
m: m=(110):(\bar{T} 10)={ }^{*} 97014^{\prime} & - \\
m: c=(110):(001)={ }^{*} 78 \quad 40 & - \\
c: \omega=(001):(T 11)=* 46 \quad 27 & - \\
b: \omega=(010):(\bar{T} 11)=56 \quad 9 & 56019^{\prime} \\
m: \omega=(110):(\bar{T} 11)=7458 & 75 \mathbf{5}
\end{array}
$$

Farblos, die meisten Krystalle waren trube, doch fanden sich auch einzelne durchsichtige. Die optische Axenebene ist zur Symmetrieebene senkrecht, geht durch den stumpfen Winkel der krystallographischen Axen $a$ und $c$ und bildet mit $(100)$ einen Winkel von etwa $82^{\circ}$. Durch das Orthopinakoid sieht man beide Axen, doch wegen der schlechten Ausbildung dieser Fläche nicht sehr gut. Der optische Axenwinkel in Luft wurde zu $65^{0}$ geschätzt. 


\section{Hexahydroterephtalsäuredimethylester.}

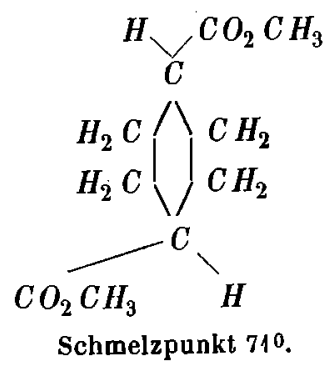

Krystallsystem: As ymmetrisch.

$$
\begin{array}{ll}
b: c=(010):(001)=69^{\circ} 0^{\prime} & \alpha=98^{0} 30^{\prime} \\
a: c=(100):(001)=4947 & \beta=12625 \\
a: b=(100):(010)=67 \quad 1 & \gamma=10250
\end{array}
$$

Grosse Tafeln, welche eine Combination der drei Pinakoide darstellen (Fig. 10). Trotz mehrfachen Umkrystallisirens aus verschiedenen Lösungsmitteln konnten keine Krystalle mit einer anderen Fläche erhalten werden, so dass das Axenverbulttniss nicht zu bestimmen war.

Farblos, durchsichtig. Auf (100) sieht man eine optische Axe unter einem Winkel von etwa $20^{\circ}$ austreten.

Fig. 10.

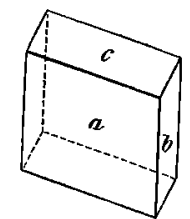

\section{Hexahydroterephtalsäurediphenylester.}

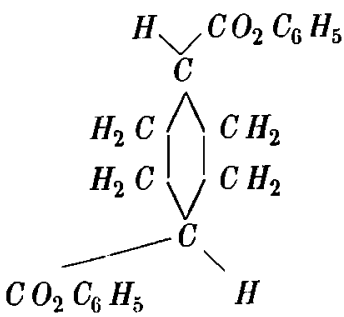

Schmelzpunkt 1540. Krystalle aus Aceton.

Krystallsystem: M on osymmet r is ch.

Fig. 11.

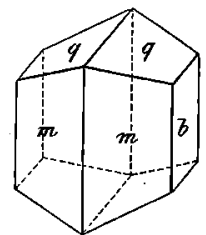

$$
\begin{aligned}
& \text { Gemessen: } \\
& b: m=(040):(110)={ }^{* 64}{ }^{\circ} 50^{\prime} \\
& \begin{array}{l}
b: m=(010):(110)={ }^{*} 645 \\
b: q=(010):(011)={ }^{*} 71 \quad 16
\end{array}
\end{aligned}
$$

Beobachtete Formen : $b=\{010\} \infty R \infty, m=\{110\}$ $\infty P, q=\{011\} R \infty$. Meist kurzprismatisch; manchmal Tafeln nach der Symmetrieebene (Fig. 11). 


$$
\begin{array}{lcc} 
& \text { Gemessen : } & \text { Berechnet : } \\
m: q=(110):(011)={ }^{*} 69^{0} 5^{\prime} & - \\
m: q=(110):(0 T 1)=852 & 85^{0} 11^{\prime}
\end{array}
$$

Farblos, schön durchsichtig. Stark lichtbrechend. Die optische Axenebene ist zur Symmetrieebene senkrecht. Auf (010) bildet die Auslöschungsrichtung mit Kante $(010):(110)$ oben einen Winkel von 521 ${ }^{\circ}$. Doppelbrechung ziemlich schwach.

Spaltbarkeit nicht beobachtet.

\section{0. $A^{1,4}$-Dihydroterephtalsäuredimethylesterdibromid.}

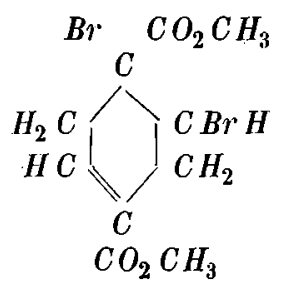

Schmelzpunkt $90^{\circ}$. Krystalle aus Holzgeist.

Krystallsystem: Monos y mmetris ch.

$$
a: b: c=1,0152: 1: 1,1641 ; \quad \beta=60^{\circ} 50^{\prime} \text {. }
$$

Fig. 12.

Beobachtele Formen : $m=\{110\} \infty P, c=\{001\} 0 P$,

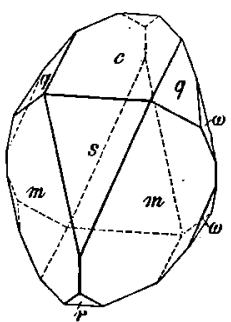
$r=\{\overline{101}\}+P \infty \infty, q=\{011\} R \infty, s=\{201\}-2 P \infty$, $\omega=\{131\}+3 R 3$ (Fig. 12). $r$ und $\omega$ treten oft gegen die anderen Flächen zurïck oder fehlen auch wohl gănzlich.

Sämmtliche Flächen waren vielfach gebrochen und gestreift und gaben daher am Goniometer schlechte Bilder. Es mussten, um einigermassen genaue Resultate zu erbalten, zehn Krystalle durchgemessen werden. Die Subslanz war vorher, um sie zu reinigen, dreimal aus Ligroin fractionirt krystallisirt worden; trotzdem scheint sie noch mit einer - durch die Analyse allerdings nicht nachweisbaren - Spur von dem Tetrabromid verunreinigt gewesen zu sein.

$$
\begin{aligned}
& \text { Gemessen : Berechnet : } \\
& m: m=(110):(110)={ }^{*} 83^{0} 5^{\prime} \quad- \\
& \text { c: } q=(001):(011)={ }^{4} 4528- \\
& c: s=(001):(201)=* 4324 \quad- \\
& c: m=(001):(110)=69 \text { ca. } \quad 68^{0} 36^{\prime} \\
& c: r=(001):(T 01)=65-67^{0} \quad 6615 \\
& \vartheta: s=(\overline{1} 01):(201)=108-111 \quad 10939 \\
& r: q=(\bar{T} 01):(011)=72 \frac{1}{2}{ }^{2} \text { ca. } 7335 \\
& m: s=(110):(201)=44.039^{\prime} \quad 4426
\end{aligned}
$$




$$
\begin{aligned}
& \text { Gemessen : Berechnet : } \\
& m: s=(011):(201)=59^{0} 36^{\prime} \quad 59^{\circ} 21^{\prime} \\
& m: r=(\bar{T} 10):(\bar{T} 01)=63 \quad 5 \quad 63 \quad 11 \\
& s: \omega=(201):(131)=9633 \quad 9631 \\
& m: \omega=(\overline{1} 10):(\bar{T} 31)=38 \quad 49 \quad 39 \quad 3 \\
& s: \omega=(201):(\overline{1} 31)=3944 \quad 3959
\end{aligned}
$$

Eine oplische Untersuchung konnte nicht angestellt werden, da die Kryställchen trübe und zu klein waren.

\section{1. $\Delta^{2,5}$-Dihydroterephtalsäaredimethylesterdibromid.}

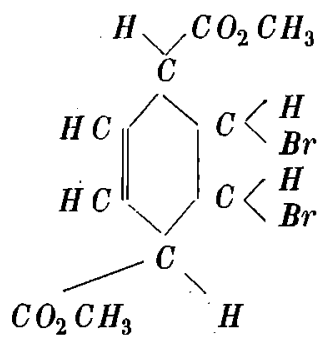

Schmelzpunkt 1100. Krystalle aus Essigäther.

Krystallsystem: Monosymmetrisch.

$$
a: b: c=1,5055: 1: 1,2218 ; \quad \beta=66^{\circ} 37^{\prime} \text {. }
$$

Beobachtete Formen: $r=\{101\}-P \infty, m=\{110\} \infty P$, $\varrho=\{\bar{T} 01\}+P \infty$. Tafeln nach dem negativen Hemidoma (Fig. 13).

$$
\begin{aligned}
& \text { Gemessen: Berechnel: }
\end{aligned}
$$

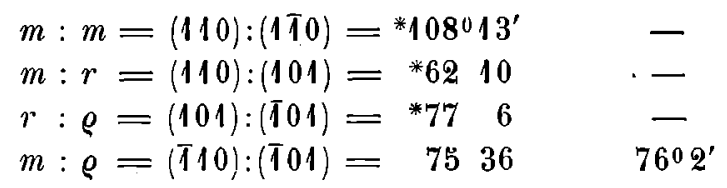

Fig. 13.

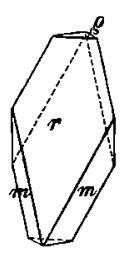

Farblos, durchsichtig. Die optische Axenebene ist auf der Symmetrieebene senkrecht und zwar gebt die erste Mittellinie dieser Fläche parallel; doch sieht man durch die vorherrschende Fläche (101) keine Axen, sondern nur einen Theil des Lemniscatensystems, da die Bisectrix mit der Normalen zu (101) einen zu grossen Winkel bildet.

Doppelbrechung ziemlich stark. 


\section{2. $\Delta^{1,4}$-Dihydroterephtalsäuredimethylestertetrabromid.}

Schmelzpunkt ca, $150,0,5$ Krystalle aus Holzgeist.

Fig. 14.

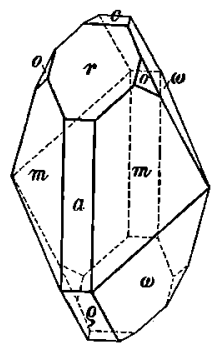

Krystallsystem : Mon osymmetrisch.

$$
\begin{gathered}
a: b: c=1,2356: 1: 0,91700 \\
\beta=81^{\circ} 54^{\prime} .
\end{gathered}
$$

Beobachtete Formen: $m=\{110\} \infty P, a=\{100\}$ $\infty P \infty, r=\{101\}-P \infty, c=\{001\} 0 P, \varrho=\{\bar{T} 01\}+P \infty$, $o=\{121\}-2 R 2, \omega=\{121\}+2 R 2$ (Fig. 14). Gewöhnlich herrschen $m, r$ und $a$ vor; manchmal sind auch alle Flächen fast gleich stark ausgebildet und etwas gerundet, die Krystalle sehen dann fast wie Glasperlen aus.

Gemessen : Berechnet:

$$
\begin{aligned}
& a: m=(100):(110)={ }^{*} 50^{0} 44^{\prime} \\
& \omega: \varrho=(\bar{T} 21):(\bar{T} 01)=* 5728 \\
& r: \omega=(101):\left(\text { T21) }={ }^{*} 8057\right. \\
& r: \varrho=(101):(\overline{1} 01)=7257 \\
& a: r=(100):(101)=4.816 \\
& m: r=(110):(101)=6459 \\
& c: \varrho=(001):(\overline{1} 01)=39.19 \\
& r: o=(101):(121)=53 \cdot 34 \\
& m: \omega=(140):(\overline{1} 21)=6134
\end{aligned}
$$

Farblos, durchsichtig, stark lichtbrechend, ausgesprochener Glasglanz. Zur optischen Untersuchung waren die Kryställchen zu klein, die Auslöschung auf den Flächen der Hemidomenzone entspricht der Symmetrie des monosymmetrischen Systems. 
13. $\Delta^{2,5}$.Dihydroterephtalsäuredimethylestertetrabromid.

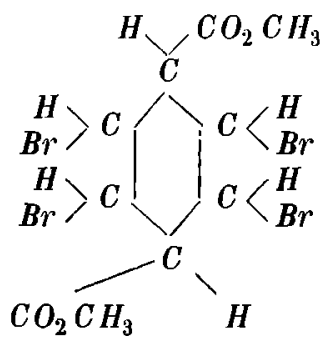

Schmelzpunkt $98^{0}$.

$a: b: c=1,3412: 1: 0,5906 ; \quad \beta=82^{0} 3^{\prime}$.

Fig. 15.

Beobachtete Formen: $c=\{001\} 0 P, m=\{110\} \infty P$, $q=\{011\}$ Roo. Tafeln nach der Basis (Fig. 15); selten sind die Krystalle kurzprismatisch nach der Verticalaxe

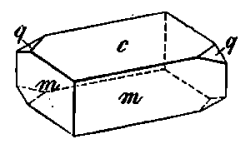
ausgebildet.

$$
\begin{aligned}
& \text { Gemessen: Berechnet: } \\
& m: m=(110):(1 \bar{T} 0)={ }^{* 106^{0}} 3^{\prime} \\
& m: c=(110):(001)={ }^{*} 8514 \\
& q: q=(011):(0 \overline{1} 1)={ }^{*} 6039 \\
& m: q=(110):(011)=6125 \\
& m: q=(110):(0 \overline{1} 1)=7056 \\
& 61^{\circ} 38^{\prime} \\
& 70 \quad 38
\end{aligned}
$$

Farblos, durchsichtig. Die optische Axenebene ist auf der Symmetrieebene senkrecht; die erste Bisectrix verläuft dieser Fläche parallel und tritt unter einem Winkel von nicht ganz $10^{\circ}$ durch die Basis aus. Der Winkel der optischen Axen in Glas für Natriumlicht beträgt etwa $68^{\circ}$; man sieht also durch (001) das Bild der beiden optischen Axen, welches, wie die Theorie es erfordert, sehr schön horizontale Dispersion zeigt.

Doppelbrechung nicht sehr stark; $\varrho<v$.

Spaltbarkeit nicht beobachtet.

\section{4. $\Delta^{1}$ - Tetrahydroterephtalsäuredimethylesterdibromid.}

$$
\begin{aligned}
& \mathrm{Br} \mathrm{CO}_{2} \mathrm{CH}_{3} \\
& \mathrm{H}_{2} \mathrm{C} \bigwedge_{\mathrm{H}_{2} \mathrm{C}} \mathrm{CBrH}_{\mathrm{C} \mathrm{H}_{2}} \\
& \mathrm{CO}_{2} \mathrm{CH}_{3} \mathrm{H},
\end{aligned}
$$

Schmelzpunkl 810. Kryslalle aus Holzgeist

Krystallsystem: Asymmetrisch. 


$$
\begin{array}{cl}
a: b: c=1,1307: 1: 1,0699 . \\
A=81052^{\prime} & \alpha=107043^{\prime} \\
B=5249 & \beta=12957 \\
C=99 \quad 19 & \gamma=7143
\end{array}
$$

Fig. 16. Beobachtete Formen: $m=\{110\} \infty P_{r}^{\prime}, \quad n=\{110\}$

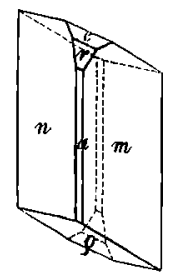
$\infty, P, a=\{100\} \infty \breve{P} \infty, r=\{101\}^{\prime} \breve{P}^{\prime} \infty, c=\{001\} 0 P$, $\varrho=\{\bar{T} 01\}, \breve{P}, \infty$ (Fig. 16). $\{110\}$ und $\{\bar{T} 01\}$ herrschen meistens vor, doch zeigten gerade diese beiden grössten Flächen oft Störungen in der Ausbildung, weshalb auch die Controlwinkel ziemlich grosse Abweichungen von den berechneten Werthen zeigen. $\{100\}$ tritt nur als schmale Abstumpfung auf. Von den der Rechnung zu Grunde gelegten Winkeln ist jeder an sechs verschiedenen Krystallen bestimmt worden; die einzelnen Werthe zeigten Abweichungen bis zu $1^{0}$.

$$
\begin{aligned}
& \text { Gemessen: Berechnet: }
\end{aligned}
$$

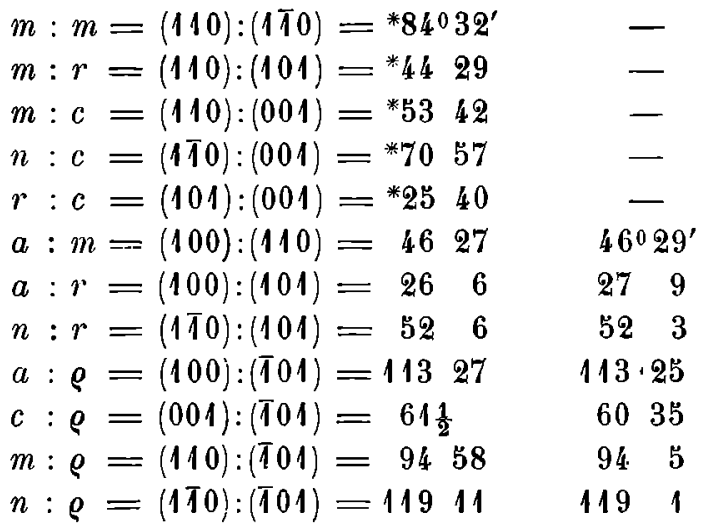

Farblos, durchsichtig. Auf (110) bildet die Auslöschungsrichtung mit Kante (110): $(1 \overline{1} 0)$ oben einen Winkel von ca. $64 \frac{1}{2}^{0} ;$ die optische Axenebene geht dieser Richtung parallel, und bildet mit der Fläche (110) nach unten einen Winkel von ca. 48 ${ }^{\circ}$. Doppelbrechung und Lichtbrechung ziemlich stark.

Spaltbarkeit nicht beobacbtet.

Die zuletzt beschriebenen fünf Substanzen lassen sich in zwei Gruppen eintheilen; die erste davon umfasst das Di- und Tetrabromid des $\Delta^{1,4}$ - Dihydroterephtalsäuredimethylesters, sowie das Dibromid des $A^{1}$-Tetrahydroesters; sie zeichnet sich dadurch aus, dass sich Bromatome an die carboxylirten Kohlenstoffatome addirt haben, was bei der zweiten Gruppe, das $\Delta^{2,5}$-Dihydro-, Di- und Tetrabromid umfassend, nicht der Fall ist. 
Die drei Glieder der ersten Gruppe zeigen morphotropische Beziehungen zu einander, die ich nicht unerwähnt lassen will. Am besten kommen dieselben zum Ausdrucke durch die Axenverhältnisse, welche ich zum Vergleiche noch einmal nebeu cinander stelle:

$$
\begin{array}{lccc} 
& a & b & c \\
\Delta^{1,4} \text {-Dihydrobromid } & 1,0125: 1: 1,1641 \\
\Delta^{1} \text { - Tetrahydrodibromid } & 1,1307: 1: 1,0699 \\
\Delta^{1,4} \text {-Dihydrotetrabromid } & 1,2356: 1: 0,9170
\end{array}
$$

Der Winkel der Axenebenen $B$ des Dibydroestertetrabromids $=81^{\circ} \mathbf{5} \mathbf{4}^{\prime}$ ist fast genau gleich $\operatorname{dem} A$ des Tetrahydroesterdibromids $=81052^{\prime}$; auch ist eine bemerkenswerthe Uebereinstimmung in den Winkeln zwischen dem letzteren und dem Dibydroesterdibromid vorhanden. Es ist nämlich bei

A1,4-Dihydrodibromid :

$$
\begin{aligned}
& (110):(201)=4.024^{\prime} \\
& (110):(1 T 0)=83 \quad 5 \\
& (110):(001)=6836
\end{aligned}
$$

$\triangle 1$-'Tetrahydrodibromid :

$$
\begin{aligned}
& (110):(101)=44029^{\prime} \\
& (110):(1 T 0)=8432 \\
& (1 \bar{T} 0):(001)=7057
\end{aligned}
$$

\section{5. (1)- Monobromhexahydroterephtalsäuredimethylester.}

$$
{ }_{\mathrm{CO}_{2} \mathrm{CH}_{3} \mathrm{CH}}^{\mathrm{Br}} \bigwedge_{\mathrm{H}_{2} \mathrm{C}}^{\mathrm{CO}_{2} \mathrm{CH}_{3}}
$$

Schmelzpunkt 700-710. Krystalle aus Ligroin bei niederer Temperatur. I. c. 182.

Krystallsystem: Monosymmetrisch.

$$
\begin{gathered}
a: b: c=0,34742: 1: 0,46944 \\
\beta=82^{\circ} 50^{\prime} .
\end{gathered}
$$

Beobachtete Formen: $c=\{001\} 0 P, r=$ $\{\bar{T} 01\}+P \infty, \quad b=\{010\} \infty R \infty, \omega=\{111\}$ $+P, \quad o=\{111\}-P, q=\{011\} R \infty ; s=$

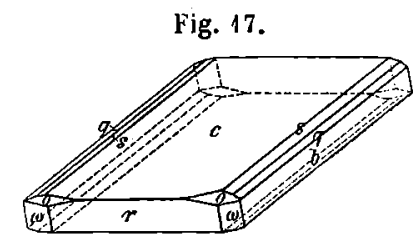
$\{012\} \frac{1}{2} R \infty$. Tafeln nach der Basis; die Klinodomen und die negative Hemipyramide treten nur schmal auf (Fig. 17).

$$
\begin{array}{llc} 
& \text { Gemessen : } & \text { Berechnet : } \\
c: r=(001):(T 01)={ }^{* 58012} & - \\
\omega: r=(111):(101)={ }^{*} 1627 & - \\
c: 0=(001):(111)={ }^{*} 5032 & -
\end{array}
$$




$\begin{array}{llr} & \text { Gemessen : } & \text { Berechnet: } \\ c: \omega=(001):(111)=59^{0} 39^{\prime} & 59^{0} 39^{\prime} \\ c: s=(001):(012)=138 & 13 \quad 7 \\ s: q=(012):(011)=1143 & 1151 \\ o: \omega=(111):(T 11)= & - & 10137\end{array}$

16. (2)-Monobromhexahydroterephtalsäaredimethylester.

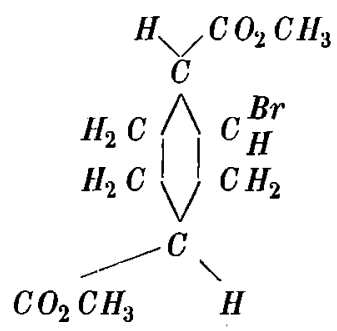

Schmelzpunkt 940-950. Krystalle aus Aether.

Krystallsystem : Mon os y m metris ch.

$$
a: b: c=2,9050: 1: 1,3694 ; \quad \beta=82049^{\prime} .
$$

Fig. 18.

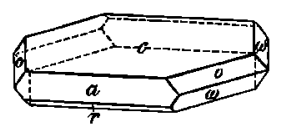

Beobachtete Folmen: $c=\{001\} 0 P, a=\{100\}$ $\infty P \infty, 0=\{111\}-P, \omega=\left\{T_{11}\right\}+P, r=\left\{T_{01}\right\}$ $+p \infty$. Tafeln nach der Basis (Fig. 18); $c$ ist meistens gekrummt.

$$
\begin{aligned}
& \text { Gemessen: Berechnet: } \\
& a: o=(100):(111)={ }^{*} 70^{0} 29^{\prime} \\
& o: \omega=(111):(111)={ }^{*} 319 \quad- \\
& \omega: \omega=(\text { T11 }):(\text { TT1 })=* 10434 \quad- \\
& a: c=(100):(001)=83 \text { appr. } \quad 82049^{\prime} \\
& a: r=(\bar{T} 00):(\bar{T} 01)=\begin{array}{lll}
7034 & 7045
\end{array} \\
& c: \omega=(001):(T 11)=5640 \quad 5647 \\
& 0: \omega=(111):(\text { TT1 })=11020 \quad 11024 \\
& o: o=(111):(1 T 1)=\begin{array}{llll}
99 & 2 & 99 & 8
\end{array}
\end{aligned}
$$

Farblos, durchsichtig; schwache Doppelbrechung. Die optische Axenebene ist der Symmetrieebene parallel; durch (001) kein Axenaustritt; die Interferenzfigur war nur im monochromatischen Lichte sichtbar. 
17. (Z) - Monobromhexahydroterephtalsäurediphenylester.

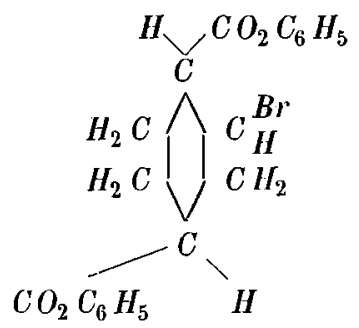

Schmelzpunkt 1240. Krystalle aus Acelon.

Krystallsystem : Monosymme trisch.

$$
a: b: c=0,4837: 1: 0,3709 ; \quad \beta=72^{03} 1^{\prime} .
$$

Beobachtete Formen : $b=\{010\} \infty \mathbb{R} \infty, q=\{011\}$ $R \infty, m=\{110\} \infty P$. Meist Tafeln nach der Symmetrieebene, seltener herrscht das Klinodoma vor; immer nach der. Klinoaxe verlängert (Fig. 19). Trolz mehrfachen Umkrystallisirens zeigten die Krystalle sämmtlich Unregelmässigkeiten in der Ausbildung; die zu einander gehörigen Flächen waren nämlich niemals parallel, obwohl dieselben schöne Reflexe gaben und die Kryställchen ganz einheit-

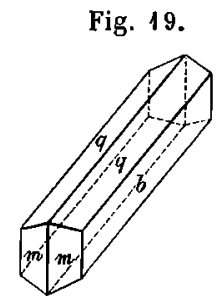
lich und gut durchsichtig waren.

$$
\begin{aligned}
& \begin{array}{l}
\text { Zahl der Grenzwerthe: Mittel: Berechnel: } \\
\text { Messungen: }
\end{array}
\end{aligned}
$$

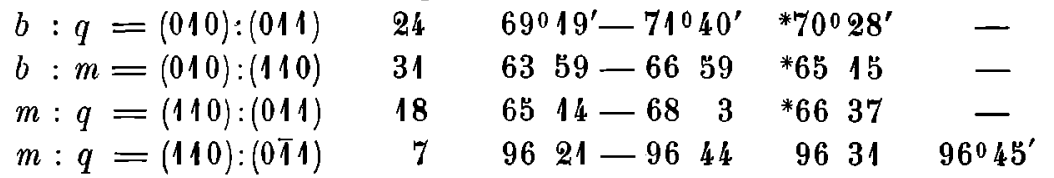

Farblos, durehsichtig. Die Schwingungsrichtung bildet auf der Symmetrieebene mit Kante (010):(011) einen Winkel von etwa $43^{\circ}$.

18. (1,4) - Dibromhexahydroterephtalsäuredimethylester.

$$
{ }_{\mathrm{H}_{2} \mathrm{C}} \bigwedge_{\mathrm{C}}^{\mathrm{Br}} \bigwedge_{\mathrm{CO}_{2} \mathrm{CH}_{3} \mathrm{Br}}^{\mathrm{CO}_{2} \mathrm{CH}_{2}} \mathrm{CH}_{3}
$$

Schmelzpunkt 1500. Krystalle aus Holzgeist. I. c. 176.

Krystallsystem: Mon osymmetrisch. 


$$
a: b: c=2,2742: 1: 0,8928 ; \quad \beta=87054^{\prime} .
$$

Beobachtele Formen: $a=\{100\} \infty \mathbb{P} \infty, m=\{110\} \infty P, n=\{610\}$ $\infty P 6, c=\{001\} 0 P, o=\{111\}-P, r=\{201\}-2 P \infty$.

Fig. 20.

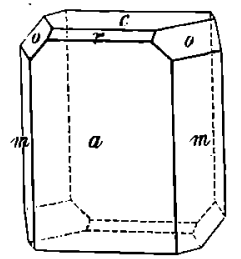
Prisma und Orthopinakoid meistens vorherrschend (Fig. 20); doch wurden auch namentlich mikroskopische Krystalle beobachtet, die mehr tafelförmig nach der Basis ausgebildet waren.

$$
\begin{aligned}
& \text { Gemessen : Berechnet: } \\
& \begin{array}{l}
a: c=(100):(001)={ }^{*} 87054 \\
a: m=(100):(110)=* 66 \quad 15
\end{array} \\
& a: 0=(100):(111)=* \text { *72 } 14 \\
& a: n=(100):(610)=2054 \\
& 20^{\circ} 45^{\prime} \\
& m: c=(110):(001)=8911 \\
& 89 \quad 9 \\
& m: 0=(110):(111)=4522 \\
& 45 \quad 18 \\
& m: o=(110):(1 T 1)=11716 \\
& 11714 \\
& m: r=(110):(201)=7514 \\
& 75 \quad 11 \\
& o: o=(111):(1 \overline{1} 1)=7837 \\
& a: r=(100):(201)= \\
& 78 \quad 44 \\
& 50 \quad 34
\end{aligned}
$$

Die Krystalle sind farblos, durchsichtig und stark lichtbrechend. Die Doppelbrechung ist nur schwach, sie zeigen daher zwischen gekreuzten Nicols Interferenzfarben. Erwärmt man ein auf der Basis liegendes Blältchen unter dem Lehmann' schen Mikroskope, so verändert sich die Interferenzfarbe auffallend schnell und durchläuft in kurzer Zeit die Farbenscala vom Roth bis zum Violett; beim Erkalten tritt das Umgekehrte ein. Die Aetherelasticität verändert sich also beim Erwärmen in der Richtung der Verticalaxe sehr stark.

19. (2,5) - Dibromhexahydroterephtalsäuredimethylester.<smiles>COC(=O)[C@H](Br)[C@H](Br)[C@H](Br)[C@H](C)OC</smiles>

Schmelzpunkt 1660. Krystalle aus Essigälher.

Krystallsystem: Monos ymmmetrisch.

$$
a: b: c=1,5477: 1: 3,0027 ; \quad \beta=85017^{\prime} \text {; }
$$


Beobachtete Formen: $c=\{001\} 0 P, a=\{100\} \infty \mathbb{P} \infty, o=\{111\}-P$, $\omega=\{111\}+P$. Tafeln nach der Basis (Fig. 21); in ihrem Habitus gleichen die Krystalle auffallend dem (2)-Monobromhexahydroäther.

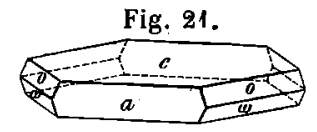

$$
\begin{aligned}
& \text { Gemessen: Berechnet: } \\
& a: o=(100):(111)={ }^{* 57020^{\prime}} \quad \text { - } \\
& o: \omega=(111):(T 11)={ }^{*} 6310 \quad- \\
& \begin{array}{l}
\omega: \omega=(\bar{T} 11):(\bar{T} 1)=* 10940 \quad- \\
o: o=(111):(1 T 1)=-106 \quad 0 \quad 106^{0} 1^{\prime}
\end{array} \\
& o: \omega=(111):(\text { T11 })=14838 \quad 148 \quad 42 \\
& c: \dot{\omega}=(001):(\bar{T} 11)=7645 \quad 7644 \\
& a: c=(100):(001)=8511 \quad 8517
\end{aligned}
$$

Farblos, durchsichtig. Die optische Axenebene steht auf der Symmetrieebene senkrecht; die erste Mittellinie geht durch den stumpfen Winkel der krystallographischen Axen $a$ und $c$. Der optische Axenwinkel ist nicht sehr gross, die Doppelbrechung ziemlich schwach.

Ebenso wie die.Bromadditionsproducte lassen sich auch die zuletzt beschriebenen Substanzen in zwei Gruppen eintheilen; in dem (1)-Monound in dem (1,4)-Dibrombexahydroester sitzen die Bromatome an den carboxylirten Kohlenstoffen, während sie in dem (2)-Mono- und in dem $(\mathbf{2}, \mathfrak{5})$-Dibromester mit nicht carboxylirten Kohlenstoffatomen verbunden sind. Während zwischen den zuerst erwähnten eine Aehnlichkeit nicht zu erkennen ist, zeigen die letzteren morphotropische Beziehungen. Dies tritt schon im Habitus hervor (siehe Figg. 18 und 21); stellt man die Krystalle einer der Substanzen anders, indem man die $a$ - und $c$-Axe vertauscht, so ergiebt sich auch eine gute Uebereinstimmung in den Axenverhältnissen:

(2) - Monobrommethylester $a: b: c=2,9050: 1: 1,3694 ; \beta=82^{04} 9^{\prime}$ (2,5)-Dibrommethylester $\quad c: b: a=3,0027: 1: 1,5477 ; \beta=8517$

Interessanter als diese Beziehungen ist ein anderer Umstand; der Phenylester der (2)-Monobromhexahydroterephtalsäure steht nämlich dem Phenylester der Hexahydroterephtalsäure in krystallographischer Beziehung so nahe, dass die beiden Substanzen als isomorph zu bezeichnen sind:

$$
\begin{array}{llll}
\text { Monobromester } & a: b: c=0,4837: 1: 0,3709 ; & \beta=72031^{\prime} \\
\text { Hexahydroester } & a: b: c=0,48619: 1: 0,35093 ; \beta=75 \quad 6
\end{array}
$$

Auch in den Formen slimmen die Krystalle itberein, indem an beiden die Symmetrieebene, Prisma und Klinodoma beobachtet wurden, während im Habitus allerdings einige Verschiedenheit zu erkennen ist (siehe Figg. 11 und 19). 
Die erwähnle Isomorphie ist nun deshalb von Interesse, weil sie ein gutes Beispiel für den Satz liefert, dass in einer organischen Verbindung bei der Substitution eines Wasserstoffes durch ein anderes Element die Krystallform um so weniger geändert wird, je grösser das Molekull ist. Die Methylester der beiden obigen Säuren (empirische Formeln $C_{10} H_{16} O_{2}$ und $\mathrm{C}_{10} \mathrm{H}_{15} \mathrm{Br} \mathrm{O}_{2}$ ) zeigen nämlich gar keine krystallographischen Beziehungen, während die Phenylester, wie wir sahen, isomorph sind. Ich babe Aebnliches schon fruher beobachtet*); Hydrobromcinchen und Ginchendibromid, die sich gerade wie Hexabydroterephtalsäureester und Monobromhexahydroterephtalsäureester durch ein Atom Brom unterscheiden, sind ebenfalls isomorph. Will man also systematisch die Aenderungen in der Krystallform organischer Substanzen verfolgen, welche durch Substitution hervorgerufen werden, so wird man gut thun, zunächst. Substanzen von grossem Molekül zu untersuchen, bei einfachen Säuren der Fettreihe z. B. Phenylester oder die Ester substituirter Phenole.

\section{Tribromlactonmethylester der Hexahydroterephtalsäure.}

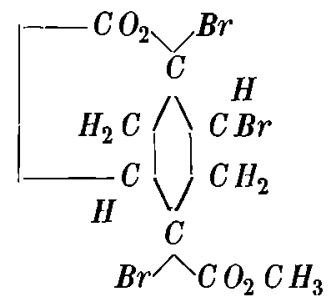

Schmelzpunkt 1870—1880. Krystalle aus Holzgeist.

Kryslallsystem: Monosymmetrisch.

$$
a: b: c=2,7026: 1: 1,9088 ; \beta=75^{0} 29 \frac{1}{2}^{\prime} .
$$

Fig. 22.

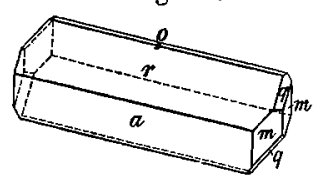

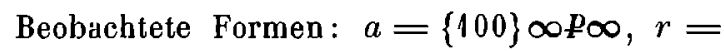
$\{101\}-P_{\infty}, \varrho=\{101\}+P \infty, m=\{110\} \infty P$ und $q=\{011\} R \infty$. Nach der Symmetrieaxe gestreckt; $\{\overline{101\}}$ tritt selten als sehr schmale Fläche auf (Fig. 22). Da mir nur wenig Material zur Verfugung stand, so konnten nur sehr kleine, etwa $\frac{1}{2} \mathrm{~mm}$ grosse Kryställchen erhalten werden. Beim Umkrystallisiren muss man verhältnissmässig viel Holzgeist anwenden, denn wenn die Krystallisation durch Abkühlen einer in der Wärme gesättigten Lösung eingeleitet wird, entstehen nur Krystalle von schaligem Aufbau, die inwendig hohl sind und nicht gemessen werden können.

*) Diese Zeitschr. 1889, 15, 392. 
Die grosse Mehrzahl der untersuchten Krystalle hatte einen durchaus asymmetrischen Habitus; es geht dies manchmal so weit, dass ein Prismenund ein Klinodomenflächenpaar ganz fehlte. Für das monosymmetrische System sprechen indessen sowohl die Winkelmessungen als auch die optischen Eigenschaften, so weit dieselben beobachtet werden konnten (gerade Auslöschung auf den Flächen der Orthozone). Ob diese Anomalie in der Ausbildung durch die Structurverhältnisse der Krystalle bedingt ist oder ob sie von einer Verunreinigung der Substanz berruhrt, kann ich nicht entscheiden.

\begin{tabular}{|c|c|}
\hline Gemessen & Berechnet: \\
\hline$a: m=(100):(110)={ }^{*} 66^{0} 5^{5}$ & - \\
\hline$a: r=(100):(101)=* 4520$ & - \\
\hline$r: q=(101):(011)=* 65$ & - \\
\hline$m: r=(110):(101)=7538$ & $75^{0} 28^{\prime}$ \\
\hline$m: q=(110):(011)=309$ & $30 \quad 13$ \\
\hline$q: q=(011):(01 \bar{T})=5641$ & 5650 \\
\hline$a: q=(100):(011)=8312$ & 839 \\
\hline 1): $(\overline{1} 01)=$ & \\
\hline
\end{tabular}

Farblos, durchsichtig.

Spaltbarkeit nicht beobachtet. 